\title{
Increased risk of hospitalization for acute hepatitis in patients with previous exposure to NSAIDs ${ }^{\dagger}$
}

\author{
Chang-Hsing Lee ${ }^{1,2}$, Jung-Der Wang ${ }^{1,3,4}$ and Pau-Chung Chen ${ }^{1 *}$ \\ ${ }^{1}$ Institute of Occupational Medicine and Industrial Hygiene, National Taiwan University College of Public Health, Taipei, Taiwan \\ ${ }^{2}$ Department of Occupational Medicine, Ton Yen General Hospital, Hsinchu County, Taiwan \\ ${ }^{3}$ Department of Internal Medicine, National Taiwan University Hospital, Taipei, Taiwan \\ ${ }^{4}$ Department of Environmental and Occupational Medicine, National Taiwan University Hospital, Taipei, Taiwan
}

\section{SUMMARY}

Background Epidemiological studies related to hospitalization due to the hepatotoxicity of traditional non-steroidal anti-inflammatory drugs (NSAIDs) are infrequent, and case reports of hepatotoxicity of nimesulide, celecoxib, and rofecoxib seem to be increasing. The reimbursement database of National Health Insurance (NHI) in Taiwan provided an opportunity for post-marketing surveillance. We conducted this study to determine the association between the use of hepatoxic NSAIDs and increased hospitalizations related to acute hepatitis.

Methods We included hospitalized subjects with a major diagnosis of acute or sub-acute necrosis of liver or toxic hepatitis and excluded viral and other causes of hepatobiliary diseases from the NHI database from 1 April 2001 to 31 December 2004. We applied two kinds of models to analyze by uni-directional and bi-directional case-crossover designs during the 28 days exposure periods and performed conditional logistic regression models.

Results There were 4519 cases of hospitalization relating to acute hepatitis, and the odds ratios of celecoxib, nimesulide, dicofenac, ibuprofen, and other hepatoxic NSAIDs were significantly increased. Compared with the adjusted odds ratios of other hepatoxic NSAIDs $(\mathrm{OR}=2.13,95 \% \mathrm{CI}=2.00,2.28)$, celecoxib $(\mathrm{OR}=1.92,95 \% \mathrm{CI}=1.38,2.69)$ was similar during the 28 days by our uni-directional casecrossover design.

Conclusions Our results provide evidence for an increased risk of hospitalization with acute hepatitis among hepatoxic NSAIDs including celecoxib users. Further mechanistic research is warranted in order to document celecoxib's hepatotoxicity. Copyright (C) 2010 John Wiley \& Sons, Ltd.

KEY WORDS - acute hepatitis; case-crossover design; cyclooxygenase 2 inhibitors; non-steroidal anti-inflammatory drugs

Received 24 November 2008; Revised 15 January 2010; Accepted 26 February 2010

\section{BACKGROUND}

Drug-induced liver injury (DILI) is a common cause of acute non-viral nontoxic liver failure, ${ }^{1}$ and liver damage is a major reason for withdrawal of a drug from the market. ${ }^{2}$ In France, the incidence rate of outpatient DILI amounts to fourteen cases per 100000 inhabitants, which is still considered as an underestimation because of difficulty in diagnosis. ${ }^{3}$ Given its

\footnotetext{
* Correspondence to: P.-C. Chen, Institute of Occupational Medicine and Industrial Hygiene, National Taiwan University College of Public Health, 17 Syujhou Road, Taipei 10055, Taiwan. E-mail: pchen@ntu.edu.tw

${ }^{\dagger}$ The authors have no conflicts of interest that are directly relevant to the content of this study.
}

relatively rare incidence, DILI may not be detected in clinical trials with limited numbers of subjects. Therefore, increasing numbers of cases of hepatotoxicity may emerge after starting marketing when a sufficient number of patients have been exposed to the new drug. ${ }^{4}$

The epidemiologic studies related to hospitalization due to the hepatotoxicity of traditional non-steroidal anti-inflammatory drugs (NSAIDs) are limited and should be pursued further. ${ }^{5}$ New NSAIDs, such as the cyclo-oxygenase-2 (COX-2) selective inhibitors, were recently developed for the treatment of chronic osteoarthritis and rheumatic arthritis and were considered to be free from gastrointestinal side effects. 
Recently, case reports related to hepatotoxicity seem to be increasing in frequency for nimesulide, ${ }^{6-8}$ celecoxib, ${ }^{9-12}$ and rofecoxib. ${ }^{13,14}$ However, a metaanalysis of clinical trials concluded that celecoxib has a low potential hepatotoxicity. ${ }^{15}$ Another cohort study ${ }^{16}$ and a case/non-case analysis ${ }^{17}$ seemed to result in the same conclusion.

The National Health Insurance (NHI) program in Taiwan is a universal system of compulsory health insurance. It provides coverage for more than $96.2 \%$ of the population, and the proportions of contracted medical care institutions are about $96.5 \%$ of all hospitals and $89.5 \%$ of all clinics. ${ }^{18}$ The NHI database contains virtually all of the health insurance, medical and prescription records for almost all citizens in Taiwan, which provides an opportunity for the postmarketing surveillance of new drugs. This study was therefore conducted in an attempt to determine the association between the use of hepatoxic NSAIDs, COX-2 selective inhibitors and the risk of hospitalizations relating to acute hepatitis.

\section{METHODS}

\section{Data source}

The dataset was obtained from the NHI database in Taiwan. The NHI files consist of comprehensive information on all medications prescribed to all insured individuals. We utilized both the outpatient visits and admission databases, which included information on gender, date of birth, date of admission, date of discharge, dates of visits, admission diagnoses, outpatient visit diagnoses, and prescription information (e.g., names, dosages, days, and expenditures). The Ethics Review Board at the National Taiwan University College of Public Health approved this study, with strict confidentiality guidelines being closely followed in accordance with personal electronic data protection regulations.

\section{Study period and population}

Three COX-2 selective inhibitors (rofecoxib, celecoxib, and nimesulide) are commercially available in Taiwan. The NHI began to reimburse for celecoxib, rofecoxib, and nimesulide on 1 April 2001, 1 July 2001, 1 and March 2003, respectively, but rofecoxib was withdrawn from the market in October 2004 because of reports of cardiovascular events. For these reasons, we chose a study period that started on 1 April 2001 and ended on the last date in the database that we applied for, 31 December 2004. The number of people in the annual dataset for all publicly insured people ranged from 21653555 in 2001 to 22134270 in 2004.

In order to prevent any misclassification of case diagnoses, we selected the study population from all publicly insured people by using the major diagnosis at admission as the definition of each case instead of minor diagnoses of admission or any diagnoses from outpatient clinics. The diagnoses in the NHI database generally follow the International Classification of Diseases, 9th Revision (ICD-9) codes. We included only the diagnoses of acute and sub-acute necrosis of the liver (ICD-9 570) and toxic (noninfectious) hepatitis (ICD-9 573.3). We excluded patients diagnosed before admission with viral hepatitis $\mathrm{A}, \mathrm{B}, \mathrm{C}$, or other viral hepatitis (ICD-9 070.0 to 070.9), viral hepatitis B or C carriers (ICD-9 V026.1 to V026.9), hepatitis in viral and other infectious diseases classified elsewhere (ICD-9 573.1 to 573.2), cholelithiasis (ICD-9 574.0 to 574.9), chronic liver disease and cirrhosis (ICD-9 571.0 to 571.9), liver abscess and sequelae of chronic liver disease (ICD-9 572.0 to 572.8 ), chronic passive congestion of liver (ICD-9 573.0), malignant neoplasm of liver and intrahepatic bile ducts (ICD-9 155.0 to 155.2), or liver metastasis (ICD-9 230.8). Because subjects might have been admitted more than once, we selected the earliest admission date for each individual.

\section{Case-crossover design}

Since there are many determinants, or potential confounders, of acute hepatitis, we applied the casecrossover design proposed by Maclure ${ }^{19}$ as a means of controlling for factors within subjects. Thus, there was no control selection bias since each case acted as its own control. In drug safety studies, the likelihood of prescribing a new medication may change over time. ${ }^{20}$ To avoid any potential bias related to time trends, we have therefore adopted uni- and symmetrically bidirectional case crossover designs, which use the four prior and two prior-posterior symmetrical periods as controls. $^{21}$ The important consideration in this design was the overall length of the exposure time period, based on case or population history. ${ }^{22}$ To make appropriate assumptions on the latent and induction times, we searched all of the available information on adverse effects from the case reports of celecoxib, rofecoxib, and nimesulide. Given that the latency period for conventional hepatoxic drugs ranges between 5 and 90 days ${ }^{23}$ and any case occurring more than 15 day (for acute hepatocellular toxicity) or 30 days (for cholestasis) after drug withdrawal can be excluded, we decided to use 28 days as exposure windows to ensure that the treatment is not stopped 
more than 15 days before onset of hepatotoxicity. ${ }^{24}$ Information was collected on prescriptions taken during each exposure window. In addition, given that transaminase elevation in case reports usually recovered within 14 days to 4 months, 90 days was selected as the washout period. For example, four prior control periods were selected, with exposure times beginning at $118,236,354$, and 472 days prior to the date of admission. In the same way, two prior control and two later control periods were selected, beginning at 118 days and 236 days before and after the date of admission (Figure 1). In brief, there were two kinds of models to analyze by uni-directional and bi-directional case-crossover designs during the 28 days periods. After comparing the results of the two models, we selected the model with the uni-directional casecrossover design for further sensitivity analysis.

\section{Exposures of interest and covariates for adjustment}

Furthermore, we undertook a search of the Micromedex ${ }^{\circledR}$ database for drugs reported as having any connection with hepatotoxicity. ${ }^{25}$ A total of 702 generic drugs were found, and the NHI in Taiwan regularly reimbursed 270 of them. We grouped them by anatomical therapeutic chemical (ATC) code and used them for adjustment. For example, if the ATC codes were M01AB, M01AC, M01AE, M01AG, M01AX, M02AA, N02BA, and B01AC, we classified these 26 drugs as 'hepatoxic NSAIDs'; J01 as 70 'antibacterial drugs'; J04A as 5 'anti-tuberculosis drugs'; N02CA, N03AA, N03AE, N05BA, and N05CD as 14 'benzodiazepine and barbiturate drugs'; and the residues as 155 'other hepatoxic drugs'. Also, there were reports of hepatotoxicity from using Chinese herbal medicines. ${ }^{26,27}$ Therefore, prescriptions of Chinese herbal medicines were grouped as 'Chinese herbs'.

We selected the two most frequent traditional NSAIDs (diclofenac and ibuprofen), three COX-2 selective inhibitors (celecoxib, rofecoxib and nimesulide) and other hepatoxic NSAIDs (21 hepatoxic NSAIDs, excluding the previous five drugs) to compare the odds ratios between them.
However, in order to investigate the condition of celecoxib prescription during the study period, we further observed the characteristics, prescribing frequencies and patterns of the cases that had celecoxib prescriptions in the risk period and the number of prescriptions for celecoxib taken all subjects per year. To clarify the dose-response relationship between the COX-2 selective inhibitors and hospitalization, we compared the daily doses of prescriptions on the date closest to admission and cumulative doses during the risk period.

\section{SENSITIVITY ANALYSIS AND EXTERNAL ADJUSTMENT FOR UNMEASURED CONFOUNDERS}

Finally, we carried out three sets of sensitivity analyses to test the robustness of our findings. First, if we defined 28-day risk and control periods by using a casecrossover design as mentioned before, not all subjects could be included in a 1-to-4 match uni-directional case-crossover design for lack of control periods during the study period. In addition, some individuals had further records in our study databases after admission. Furthermore, some subjects might have used celecoxib but stopped after admission. According to these different prescribing patterns, we stratified the sample according to these subgroups. Second, sex, older age, and the status of diseases may affect DILI. ${ }^{4}$ Common approved indications for treatment with celecoxib and rofecoxib were osteoarthritis (ICD-9 715) and rheumatoid arthritis (ICD-9 714.0, 714.3). Then, we explored the data for any of the following conditions or co-morbidities before admission for acute non-viral hepatitis: diabetes mellitus (ICD-9 250), essential hypertension (ICD-9 401), obesity and hyperlipidemia (ICD-9 272, 278), chronic kidney disease and renal failure (ICD-9 585 to 586), hyperthyroidism (ICD-9 242), fasting and malnutrition (ICD-9 260 to 263), or neoplasms (ICD-9 140 to 239). Pregnancy (ICD-9 646.7, V72.40 to 72.42 , V22.0 to 22.2) was also considered for 300 days before admission. We stratified the total population into

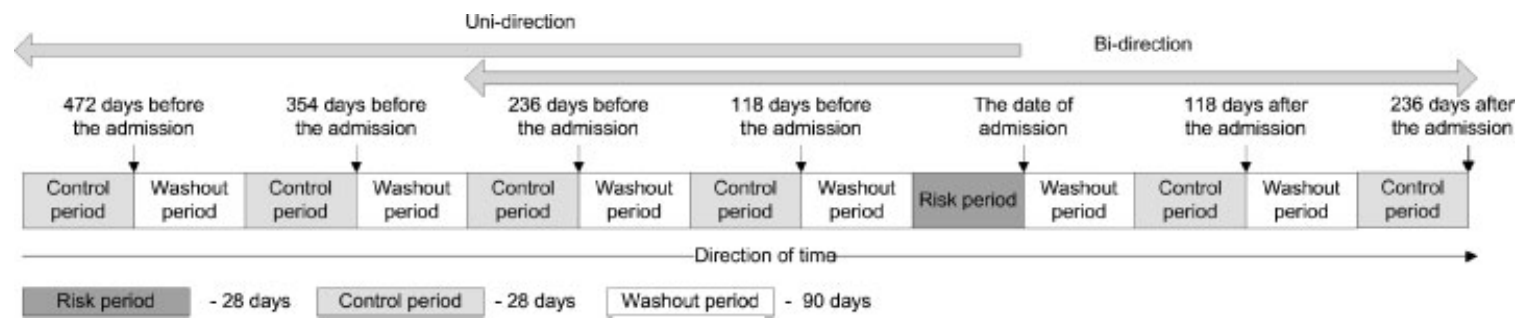

Figure 1. Timeline of the risk and four uni- and bi-directional control periods 
subgroups according to the three co-morbidities that were diagnosed most frequently. Third, we grouped the hepatoxic drugs into classes to adjust for the fact that some specific drugs within a class might be hepatoxic. We also stratified them into subgroups according to the seven most frequent co-prescriptions that were used by our subjects during the study period.

\section{Data analysis}

Since the design of this study utilized one case period matched with four control periods, we analyzed the data through construction of conditional logistic regression models to explore the association between hospitalization and prescriptions while controlling for antibiotics, anti-tuberculosis drugs, benzodiazepines, and barbiturates, Chinese herbs and other hepatoxic drugs. We then calculated odds ratios and $95 \%$ confidence intervals (CIs). The analysis of the data was performed using SAS version 9.13 software (SAS Institute Inc., Cary, NC).

\section{RESULTS}

From the data on all insured individuals from the Taiwan NHI database between 1 April 2001 and 31 December 2004, there were a total of 15088 subjects who conformed to the inclusion criteria. Among them, 1568 admitted cases were excluded in order to allow for the risk period and at least one control period during the study period. Otherwise, based on the exclusion criteria, another 9001 subjects who were diagnosed as viral and other causes of hepatobiliary diseases were excluded. Of the remaining 4519 cases, $69.6 \%$ of the individuals were 15-64 years of age, with a mean age of $46.1 \pm 21.7$ years. If we defined 28-day time windows by using a uni-directional case-crossover design, the total numbers of subjects that could be matched for 1-to-1, 1-to-2, 1-to-3, and 1-to-4 were 416, 443,515 , and 3145, respectively. In addition, 98 individuals had no further records in either the inpatient or admission databases or were assumed to have died during the relevant hospitalization. The five most common co-morbid diseases before admission were essential hypertension (21.0\%), osteoarthritis $(14.5 \%)$, diabetes mellitus (14.0\%), neoplasms (13.9\%), and obesity and hyperlipidemia (11.1\%). There were 35,19 , and 30 subjects who had been prescribed celecoxib, rofecoxib, and nimesulide, respectively, within the 28 -day risk period, as summarized in Table 1.

Moreover, we explored 35 cases that had celecoxib prescriptions in the 28-day risk period. Their mean age was $60.9 \pm 20.0$ years, and 23 cases were females.
Table 1. Characteristics, co-morbidities, and hepatoxic co-prescriptions of study subjects with initial admission diagnosis of acute non-viral hepatitis, 2001-2004

\begin{tabular}{|c|c|c|}
\hline Characteristics & No. & $\%$ \\
\hline Total & 4519 & 100.0 \\
\hline \multicolumn{3}{|l|}{ Sex } \\
\hline Male & 2580 & 57.1 \\
\hline Female & 1939 & 42.9 \\
\hline \multicolumn{3}{|l|}{ Age } \\
\hline$<15$ years & 332 & 7.3 \\
\hline $15-64$ years & 3143 & 69.6 \\
\hline$\geq 65$ years & 1044 & 23.1 \\
\hline Co-morbidities that may enhance susceptibility & No.* & $\%$ \\
\hline Essential hypertension & 951 & 21.0 \\
\hline Osteoarthritis & 653 & 14.5 \\
\hline Diabetes mellitus & 631 & 14.0 \\
\hline Neoplasms & 626 & 13.9 \\
\hline Obesity and hyperlipidemia & 501 & 11.1 \\
\hline Chronic kidney disease and renal failure & 255 & 5.6 \\
\hline Hyperthyroidism & 80 & 1.8 \\
\hline Rheumatoid arthritis & 67 & 1.5 \\
\hline Systemic lupus erythematosus & 39 & 0.9 \\
\hline Fasting, malnutrition & 37 & 0.8 \\
\hline Pregnancy & 25 & 0.6 \\
\hline Prescriptions & No. $^{\dagger}$ & $\%$ \\
\hline Celecoxib & 35 & 0.8 \\
\hline Rofecoxib & 19 & 0.4 \\
\hline Nimesulide & 30 & 0.7 \\
\hline Diclofenac & 580 & 12.8 \\
\hline Ibuprofen & 287 & 6.4 \\
\hline Other hepatoxic NSAIDs $\ddagger$ & 1487 & 32.9 \\
\hline Co-prescriptions & No. ${ }^{\dagger}$ & $\%$ \\
\hline Chinese herbs & 261 & 5.8 \\
\hline Antibacterial drugs & 735 & 16.3 \\
\hline Anti-tuberculosis drugs & 112 & 2.5 \\
\hline Benzodiazepine and barbiturates & 499 & 11.0 \\
\hline Other hepatoxic drugs & 1687 & 37.3 \\
\hline
\end{tabular}

NSAIDs, non-steroidal anti-inflammatory drugs.

*Each subject might have any number of co-morbidities before admission. ${ }^{\dagger}$ Each subject might have any number of prescriptions and co-prescriptions during the 28-day risk period.

${ }^{\ddagger}$ Other hepatoxic NSAIDs: all hepatoxic NSAIDs except celecoxib, rofecoxib, nimesulide, diclofenac, and ibuprofen

Mean percentage of all prescription days divided by total days from 1 April 2001 to admission date was $17.5 \pm 22.2 \%$. Fifteen cases stopped celecoxib after admission. We also found that the number of prescriptions for celecoxib taken by 4519 cases per year increased from 99 to 650 between 1 April 2001 and 31 December 2004.

In Table 2, it is shown that the odds ratios of all NSAIDs significantly increased during the 28 days by uni-directional designs. The odds ratios yielded by unidirectional designs were also larger than those obtained by bi-directional designs. Compared with the adjusted odds ratios of other hepatoxic NSAIDs $(\mathrm{OR}=2.13$, $95 \% \mathrm{CI}=2.00,2.28)$, nimesulide $(\mathrm{OR}=2.63,95 \% \mathrm{CI}=$ $1.83,3.77)$ seemed slightly larger, but celecoxib's $(\mathrm{OR}=1.92,95 \% \mathrm{CI}=1.38,2.69)$ was similar by 
Table 2. Adjusted odds ratios of COX-2 selective inhibitors, diclofenac, ibuprofen, and other hepatoxic NSAIDs on hospitalizations with acute non-viral hepatitis during the 28 days of the risk period with prior and posterior control periods, 2001-2004

\begin{tabular}{|c|c|c|c|c|c|c|c|c|c|c|}
\hline & & \multirow[t]{2}{*}{ Cases $(N=4519)$} & \multicolumn{4}{|l|}{ Prior controls } & \multicolumn{4}{|c|}{ Prior and posterior controls } \\
\hline & & & $\begin{array}{l}\text { Controls } \\
(N=15427)\end{array}$ & $\mathrm{OR}^{*}$ & $95 \% \mathrm{C}$ & & $\begin{array}{l}\text { Controls } \\
(N=16670)\end{array}$ & $\mathrm{OR}^{*}$ & $95 \% \mathrm{CI}$ & \\
\hline Celecoxib & & 35 & 63 & 1.92 & 1.38 & 2.69 & 73 & 1.71 & 1.23 & 2.39 \\
\hline \multirow[t]{2}{*}{ Daily dose $e^{\dagger}$} & $\geq 200 \mathrm{mg}$ & 28 & 53 & 1.86 & 1.28 & 2.71 & 63 & 1.63 & 1.12 & 2.36 \\
\hline & $<200 \mathrm{mg}$ & 7 & 10 & 2.20 & 1.04 & 4.64 & 10 & 2.17 & 1.03 & 4.58 \\
\hline \multirow[t]{2}{*}{ Cumulative dose $\mathrm{e}^{\ddagger}$} & $\geq 2000 \mathrm{mg}$ & 25 & 51 & 1.77 & 1.19 & 2.63 & 55 & 1.65 & 1.11 & 2.45 \\
\hline & $<2000 \mathrm{mg}$ & 10 & 12 & 2.45 & 1.31 & 4.58 & 18 & 1.89 & 1.01 & 3.52 \\
\hline Rofecoxib & & 19 & 45 & 1.60 & 1.01 & 2.51 & 66 & 1.18 & 0.75 & 1.85 \\
\hline Nimesulide & & 30 & 31 & 2.63 & 1.83 & 3.77 & 42 & 2.19 & 1.53 & 3.15 \\
\hline Diclofenac & & 580 & 794 & 2.22 & 2.05 & 2.42 & 889 & 2.06 & 1.90 & 2.24 \\
\hline Ibuprofen & & 287 & 318 & 2.51 & 2.23 & 2.82 & 383 & 2.24 & 1.99 & 2.52 \\
\hline Other hepatoxic NSAIDs ${ }^{\S}$ & & 918 & 1350 & 2.13 & 2.00 & 2.28 & 1594 & 1.91 & 1.78 & 2.04 \\
\hline
\end{tabular}

COX-2, cyclo-oxygenase-2; NSAIDs, non-steroidal anti-inflammatory drugs; OR, odds ratio; CI, confidence interval.

*Adjusted for antibacterial drugs, anti-tuberculosis drugs, benzodiazepines, and barbiturates, Chinese herbs and other hepatoxic drugs.

${ }^{\dagger}$ Daily doses of prescriptions on the date closest to admission.

${ }^{\ddagger}$ Cumulative doses of prescriptions during the risk period.

${ }^{\S}$ Other hepatoxic NSAIDs: all hepatoxic NSAIDs except celecoxib, rofecoxib, nimesulide, diclofenac, and ibuprofen.

uni-directional designs. There appeared to be no significant dose-response relationship for celecoxib when we stratified the daily doses into above or below $200 \mathrm{mg} /$ day or cumulative doses into above or below $2000 \mathrm{mg}$. The number of subjects with larger daily doses or cumulative doses was too small to be stratified in the analyses of rofecoxib and nimesulide.
The sensitivity analysis for the 28-day risk period in the uni-directional design is summarized in Table 3. We did not find any apparent changes in subgroups of sex, age, different matched patterns, prescribing conditions, three common co-morbidities, or seven potentially hepatoxic coprescriptions.

Table 3. Sensitivity analysis of adjusted odds ratios between hospitalizations with acute non-viral hepatitis and celecoxib stratified by subgroups with sex, age, different matched patterns, prescribing conditions, co-morbidities, and co-prescriptions during the 28 days of the risk period with four prior control periods

\begin{tabular}{|c|c|c|c|c|c|c|}
\hline \multirow{2}{*}{$\begin{array}{l}\text { Models } \\
\text { Main model }\end{array}$} & \multirow{2}{*}{$\begin{array}{l}\text { Items } \\
\text { Total population }\end{array}$} & \multirow{2}{*}{$\begin{array}{c}\text { Cases } \\
35\end{array}$} & \multirow{2}{*}{$\begin{array}{c}\text { Controls } \\
63\end{array}$} & \multirow{2}{*}{$\begin{array}{l}\mathrm{OR}^{*} \\
1.92\end{array}$} & \multicolumn{2}{|c|}{$95 \% \mathrm{CI}$} \\
\hline & & & & & 1.38 & 2.69 \\
\hline \multirow{3}{*}{$\begin{array}{l}\text { Subgroup efrects } \\
\text { Sex }\end{array}$} & & & & & & \\
\hline & Male & 13 & 27 & 1.73 & 1.00 & 3.00 \\
\hline & Female & 22 & 36 & 2.05 & 1.35 & 3.13 \\
\hline \multirow[t]{2}{*}{ Age } & $<65$ years & 15 & 15 & 2.70 & 1.62 & 4.51 \\
\hline & $>65$ years & 20 & 48 & 1.54 & 0.99 & 2.40 \\
\hline \multirow[t]{4}{*}{ Matched patterns } & One risk to one control periods & 2 & 1 & 3.68 & 0.90 & 14.99 \\
\hline & One risk to two control periods & 0 & 1 & - & - & - \\
\hline & One risk to three control periods & 1 & 1 & 2.57 & 0.36 & 18.57 \\
\hline & One risk to four control periods & 32 & 60 & 1.88 & 1.32 & 2.67 \\
\hline \multirow[t]{2}{*}{ Prescribing conditions } & Stop celecoxib after admission & 15 & 3 & 4.60 & 2.74 & 7.74 \\
\hline & Die after admission & 4 & 6 & 2.13 & 0.79 & 5.74 \\
\hline \multirow[t]{3}{*}{ Co-morbidities ${ }^{\dagger}$} & Essential hypertension & 18 & 43 & 1.55 & 0.97 & 2.47 \\
\hline & Diabetes mellitus & 12 & 23 & 1.81 & 1.02 & 3.22 \\
\hline & Osteoarthritis & 17 & 43 & 1.50 & 0.93 & 2.42 \\
\hline \multirow[t]{7}{*}{ Co-prescriptions ${ }^{\ddagger}$} & Chlorzoxazone & 21 & 50 & 1.58 & 1.03 & 2.43 \\
\hline & Sulfamethoxazole & 23 & 37 & 2.05 & 1.36 & 3.11 \\
\hline & Amlodipine & 12 & 24 & 1.77 & 1.00 & 3.13 \\
\hline & Allopurinol & 6 & 13 & 1.69 & 0.75 & 3.79 \\
\hline & Metformin & 9 & 19 & 1.70 & 0.88 & 3.30 \\
\hline & Rifampin & 5 & 6 & 2.51 & 1.03 & 6.08 \\
\hline & Isoniazid & 3 & 4 & 2.42 & 0.77 & 7.63 \\
\hline
\end{tabular}

OR, odds ratio; CI, confidence interval.

*Adjusted for Chinese herbs and other hepatoxic medications.

${ }^{\dagger}$ Diseases may affect hepatotoxicity.

${ }^{\ddagger}$ Potential hepatoxic co-prescriptions. 


\section{DISCUSSION}

Our study found that nimesulide, dicofenac, ibuprofen, and other hepatoxic NSAIDs increased the risk of hospitalization for acute hepatitis, which corroborates previous studies. Moreover, there was a significantly higher risk in the use of celecoxib, which has never been reported before. ${ }^{15-17}$ Before drawing any conclusions, we should carefully evaluate any alternative explanations.

To prevent potential bias by misdiagnosis, we deliberately included only patients who were hospitalized and excluded other possible causes of hepatobiliary diseases, including all hepatitis related to infection, alcohol, cholelithiasis and de-compensated hepatic conditions, as these predisposed conditions might more likely lead to liver injury from potential hepatoxic drugs. Thus, our estimates were more conservative because we did not include the above cases. Since we did not have any direct access to original clinical data, our study was necessarily limited to the more severe cases resulting in hospitalizations, which undoubtedly results in underestimation of hepatotoxicity with only mild manifestations.

The higher risk during 28 days observed by unidirectional design might be related to acute hepatotoxicity of celecoxib, such as in the patients who were previously sensitized to the drugs. ${ }^{11,23}$ Otherwise, we speculate that the increased risk may be partly due to confounding by indication according to the trend by sensitivity analysis of time windows. It is usually influenced by several factors, such as physician's decision, severity of the disease, concomitant medical conditions, and therapy. ${ }^{28}$

We also used bi-directional design to reduce time trend bias of celecoxib and risk of celecoxib users was smaller but significantly high still. The risk of taking the same medication might disappear after the correct diagnosis of DILI is made. A higher odds ratio for those who had stopped taking celecoxib after admission also supported our conjecture. Thus, our bi-directional design might underestimate the true risk.

Our results also corroborate the evidence that the risk of hospitalizations for hepatopathy among users of nimesulide was higher than for those using other hepatoxic NSAIDs. ${ }^{16,17}$ On the other hand, there seemed to be a slightly higher toxicity for celecoxib in our study compared to those conducted in Western countries. ${ }^{15,29}$ Our analysis showed that the 35 cases exposed to celecoxib were much older and more commonly female than the overall case population. Besides, Table 3 reveals female patients, with less than 65 years had higher risk in this population. Moreover, these factors, with racial differences, may be associated with susceptibility ${ }^{30}$ to DILI. However, these factors should be already adjusted by the case-crossover design.

In addition, odds ratios adjusted by either daily or cumulative dose during the risk period did not show any dose-response relationship. This observation appears to conform to the findings of idiosyncratic DILI. ${ }^{31}$

We conducted further sensitivity analyses by stratification to clarify the misclassifications and potential confounders. Table 3 reveals no valuable changes in the odds ratios of the subgroups with sex, age, different prescribing conditions, matched patterns, and co-morbidities. While the medications being studied were co-prescribed with seven potential hepatoxic drugs, the results reveal that there were no dramatic contributions of drug-drug interactions.

Potential limitations of unmeasured confounders, patient compliance, and use of other out-of-pocket drugs should also be discussed. First, we applied a casecrossover design to control for unmeasured confounders, such as personal constitution and lifestyle factors. Second, although the reimbursement data used in this study cannot provide information on actual intake of prescribed medication, such a limitation usually leads toward random misclassification and an under-estimation of risk. Finally, our NHI covers comprehensively almost all kinds of medications, except for unproven new chemotherapeutic drugs; our subjects rarely paid to purchase additional medications.

Our results provide additional safety information for the use of celecoxib as well as hepatoxic NSAIDs, with the finding that there was an increased risk of hospitalization for acute hepatitis. Further mechanistic research is warranted for celecoxib's hepatotoxicity.

\section{KEY POINTS}

- The first pharmacoepidemiologic study by using the case-crossover design in the database survey the hepatotoxicity of new drugs in the real world.

- The results provide evidence for an increased risk of hospitalization with acute hepatitis among nimesulide, dicofenac, ibuprofen, and hepatoxic NSAIDs especially including celecoxib users.

- The risk of celecoxib's hepatotoxicity is higher than the results of previous studies in the western countries.

- The study provides additional safety information for the use of celecoxib. 


\section{ACKNOWLEDGEMENTS}

This study was partially supported by grants from the Department of Health (DOH95-TD-D-113-027) and National Health Research Institutes (NHRI-EX96-9204PP), Taiwan.

\section{REFERENCES}

1. Ostapowicz G, Fontana RJ, Schiodt FV, et al. Results of a prospective study of acute liver failure at 17 tertiary care centers in the United States. Ann Intern Med 2002; 137: 947-954.

2. Bakke OM, Manocchia M, de Abajo F, et al. Drug safety discontinuations in the United Kingdom, the United States, and Spain from, 1974, through 1993: a regulatory perspective. Clin Pharmacol Ther 1995; 58: 105-117.

3. Sgro C, Clinard F, Ouazir K, et al. Incidence of drug-induced hepatic injuries: a French population-based study. Hepatology 2002; 36: 451-455.

4. Larrey D. Epidemiology and individual susceptibility to adverse drug reactions affecting the liver. Semin Liver Dis 2002; 22: 145-155.

5. Rubenstein JH, Laine L. Systematic review: the hepatotoxicity of nonsteroidal anti-inflammatory drugs. Aliment Pharmacol Ther 2004; 20 : 373-380.

6. Van Steenbergen W, Peeters P, De Bondt J, et al. Nimesulide-induced acute hepatitis: evidence from six cases. J Hepatol 1998; 29: 135-141.

7. Merlani G, Fox M, Oehen HP, et al. Fatal hepatotoxicity secondary to nimesulide. Eur J Clin Pharmacol 2001; 57: 321-326.

8. Andrade RJ, Lucena MI, Fernandez MC, Gonzalez M. Fatal hepatitis associated with nimesulide. J Hepatol 2000; 32: 174.

9. Carrillo-Jimenez R, Nurnberger M. Celecoxib-induced acute pancreatitis and hepatitis: a case report. Arch Intern Med 2000; 160: 553-554.

10. Galan MV, Gordon SC, Silverman AL. Celecoxib-induced cholestatic hepatitis. Ann Intern Med 2001; 134: 254.

11. O'Beirne JP, Cairns SR. Cholestatic hepatitis in association with celecoxib. Br Med J 2001; 323: 23.

12. Nachimuthu S, Volfinzon L, Gopal L. Acute hepatocellular and cholestatic injury in a patient taking celecoxib. Postgrad Med J 2001; 77 : $548-550$.

13. Harsch IA, Michaeli P, Hahn EG, Konturek PC, Klein R. A rare case of rofecoxib-induced cholestatic hepatitis. Dig Liver Dis 2003; 35: 911912.

14. Huster D, Schubert C, Berr F, et al. Rofecoxib-induced cholestatic hepatitis: treatment with molecular adsorbent recycling system (MARS). J Hepatol 2002; 37: 413-414.
15. Maddrey WC, Maurath CJ, Verburg KM, Geis GS. The hepatic safety and tolerability of the novel cyclooxygenase- 2 inhibitor celecoxib. Am J Ther 2000; 7: 153-158.

16. Traversa G, Bianchi C, Da Cas R, et al. Cohort study of hepatotoxicity associated with nimesulide and other nonsteroidal anti-inflammatory drugs. Br Med J 2003; 327: 18-22.

17. Sanchez-Matienzo D, Arana A, Castellsague J, Perez-Gutthann S. Hepatic disorders in patients treated with COX-2 selective inhibitors or nonselective NSAIDs: a case/noncase analysis of spontaneous reports. Clin Ther 2006; 28: 1123-1132.

18. Department of Health. Health and national health insurance annual statistics in Taiwan. Available at: http://www.doh.gov.tw/statistic/ index.htm accessed [1 November 2008].

19. Maclure M. The case-crossover design: a method for studying transient effects on the risk of acute events. Am J Epidemiol 1991; 133: $144-153$.

20. Schneeweiss S, Stürmer T, Maclure M. Case-crossover and case-timecontrol designs as alternatives in pharmacoepidemiologic research. Pharmacoepidemiol Drug Saf 1997; 6: S51-59.

21. Navidi W. Bidirectional case-crossover designs for exposures with time trends. Biometrics 1998; 54: 596-605.

22. Maclure M, Mittleman MA. Should we use a case-crossover design? Annu Rev Public Health 2000; 21: 193-221.

23. Benichou C. Criteria of drug-induced liver disorders. Report of an international consensus meeting. J Hepatol 1990; 11: 272-276.

24. Danan G, Benichou C. Causality assessment of adverse reactions to drugs-I. A novel method based on the conclusions of international consensus meetings: application to drug-induced liver injuries. J Clin Epidemiol 1993; 46: 1323-1330.

25. Micromedex ${ }^{\circledR} 1.0$ Healthcare Series [Internet database]. Thomson Reuters (Healthcare) Inc, Greenwood Village, Colorado, USA. Available at: http://www.thomsonhc.com [Accessed 1 November 2008].

26. Stickel F, Patsenker E, Schuppan D. Herbal hepatotoxicity. J Hepatol 2005; 43: 901-910.

27. Lee $\mathrm{CH}$, Wang JD, Chen PC. Case-crossover study of hospitalization for acute hepatitis in Chinese herb users. J Gastroenterol Hepatol 2008; 23: $1549-1555$.

28. Signorello LB, McLaughlin JK, Lipworth L, Friis S, Sorensen HT, Blot WJ. Confounding by indication in epidemiologic studies of commonly used analgesics. Am J Ther 2002; 9: 199-205.

29. Rostom A, Goldkind L, Laine L. Nonsteroidal anti-inflammatory drugs and hepatic toxicity: a systematic review of randomized controlled trials in arthritis patients. Clin Gastroenterol Hepatol 2005; 3: 489-498.

30. Larrey D, Pageaux GP. Genetic predisposition to drug-induced hepatotoxicity. J Hepatol 1997; 26: (Suppl 2): 12-21.

31. Kaplowitz N. Idiosyncratic drug hepatotoxicity. Nat Rev Drug Discov 2005; 4: 489-499. 